\title{
Synergistic regulatory effects of microRNAs on brain glioma cells
}

\author{
YILEI ZHAO ${ }^{1 *}$, XIAOMENG CUI $^{2 *}$, WENLIANG ZHU ${ }^{3 *}$, XIN CHEN $^{4}, \mathrm{CHEN} \mathrm{SHEN}^{4}$, \\ ZHENDONG LIU ${ }^{4}$, GUANG YANG $^{4}$, YAOHUA LIU $^{4}$ and SHIGUANG ZHAO ${ }^{4}$ \\ ${ }^{1}$ Department of Pharmacy, The First Affiliated Hospital of Harbin Medical University, Harbin, Heilongjiang 150001; \\ ${ }^{2}$ Institute of Measurement-Control Technology and Communications Engineering, Harbin University of \\ Science and Technology, Harbin, Heilongiiang 150010; ${ }^{3}$ Institute of Clinical Pharmacology, The Second \\ Affiliated Hospital of Harbin Medical University, Harbin, Heilongjiang 150086; ${ }^{4}$ Department of Neurosurgery, \\ The First Affiliated Hospital of Harbin Medical University, Harbin, Heilongjiang 150001, P.R. China
}

Received April 2, 2016; Accepted March 20, 2017

DOI: $10.3892 / \mathrm{mmr} .2017 .6709$

\begin{abstract}
Glioma is among the most common types of cancer of the central nervous system and is difficult to cure. Due to the lack of glioma-specific treatments, patients with glioma exhibit high mortality rates. MicroRNAs (miRNAs) participate in the pathogenesis of glioma, and upregulation of specific miRNAs promotes cell proliferation, whereas apoptosis-inducing miRNAs are markedly downregulated in the context of glioma. Therefore, miRNAs may be important contributors to the pathogenesis of glioma. In the present study, nine miRNAs were investigated as miRNA-miRNA pairs, and the measured cell viabilities were consistent with the results of synergy predictions. Extensive synergy occurred among upregulated miRNAs in U87 cells, whereas downregulated miRNAs rarely exhibited synergism. Treatment with an miRNA-miRNA pair exhibiting strong synergy increased the inhibitory effects of these miRNAs on tumor cells, and the combined inhibitory effects were increased compared with the sum of the individual inhibitory effects of each miRNA. Using cell viability assays, TUNEL staining, and flow cytometry, the present study demonstrates that cotransfection with miR-20a and miR-21inhibitors resulted in the highest synergistic effect on the promotion of apoptosis in U87 cells. The results of the present study provide important insights into the potential use of miRNAs in the treatment of glioma.
\end{abstract}

Correspondence to: Dr Shiguang Zhao, Department of Neurosurgery, The First Affiliated Hospital of Harbin Medical University, 23 Postal Street, Harbin, Heilongjiang 150001, P.R. China

E-mail: guangsz@hotmail.com

*Contributed equally

Key words: glioma, microRNA, synergy, apoptosis, microRNA-21, microRNA-20a

\section{Introduction}

Glioma is an incurable primary malignant tumor with a high recurrence rate. The life expectancy of patients with glioma is $\sim 1$ year, and that of patients exhibiting recurrence is $\sim 4$ months (1-3). Survey results have demonstrated that the incidence of glioma has increased in recent years. The principal treatment regimen used clinically is surgical resection followed by chemotherapy (4); however, this treatment regimen is not able to cure glioma as gliocytes exhibit invasive growth with unclear boundaries from normal brain tissue, resulting in incomplete surgical resection, and may be resistant to chemotherapy $(5,6)$. Therefore, improved therapies are required to achieve improved clinical outcomes and reduce the rate of recurrence in patients with glioma.

Numerous studies have demonstrated that microRNAs (miRNAs/miRs) are extensively involved in regulating mutations in glioma-associated genes (7), cellular invasion, migration, apoptosis (8) and blood vessel formation around the residual tumor (9). Analysis of the expression levels of miRNAs in normal brain tissue compared with glioma has demonstrated that 22 miRNAs, including miR-15a, miR-16, miR-21, the miR-17-92 cluster, miR-26a and miR-221/222, are upregulated in glioma and promote the proliferation, migration and invasion of cancer cells (10-13), whereas 33 miRNAs, including miR-7, miR-137, miR-145, the miR-181 family and miR-195, are downregulated and inhibit apoptosis in cancer cells (14-16). The oncogenic miRNA miR-21 has been observed to be associated with malignant glioma, and its expression level is directly associated with tumor grade; patients with low miRNA-21 expression exhibit increased survival times and improved clinical outcomes (17).

In long-term studies, researchers have demonstrated that a single miRNA is able to regulate $>100$ proteins encoded by target genes and that approximately one-third of proteins involved in common biological activities are regulated by different miRNAs. Therefore, various miRNAs exhibit synergistic activities and are able to regulate identical target mRNAs to mediate the same biological process $(18,19)$. In a previous study, miR-1 and miR-21 were demonstrated to function synergistically during myocardial ischemia, as predicted by synergy 
score calculations and validated by cellular experiments (20). Simultaneous modulation of two miRNAs was observed to have apoptosis-inhibiting effects on cardiac myocytes and to allow for a reduction in the concentration of miRNAs in order to effectively avoid off-target effects (21).

Therefore, it was hypothesized that miRNAs may act synergistically during the regulation of proliferation and apoptosis in glioma. The present study aimed to identify glioma-sensitive miRNA combinations with potential synergistic regulatory effects based on synergy scores and to validate such miRNA combinations at the cellular level, in order to provide a rational, effective, novel noncoding RNA-based treatment strategy for the prevention and treatment of glioma.

\section{Materials and methods}

Collection of patient tissues. The present study was performed with the ethical approval of the Human Ethics Committee of The First Affiliated Hospital of Harbin Medical University (Harbin, China) in accordance with the Declaration of Helsinki, and written informed consent was obtained from all enrolled patients. Noncancerous brain tissues were collected from the temporal lobes of four patients with epilepsy. All tissues were frozen in liquid nitrogen immediately following surgical removal and stored at $-80^{\circ} \mathrm{C}$.

Cell culture and transfection. U87 cells, obtained from the Cell Resource Center of the Shanghai Institute of Life Sciences (Chinese Academy of Sciences, Shanghai, China), were cultured in high-glucose Dulbecco's modified Eagle's medium (Invitrogen; Thermo Fisher Scientific, Inc., Waltham, MA, USA) with $10 \%$ fetal bovine serum (Invitrogen; Thermo Fisher Scientific,Inc.) and $100 \mathrm{U} / \mathrm{ml}$ streptomycin/penicillin (Beyotime Institute of Biotechnology, Haimen, China) at $37^{\circ} \mathrm{C}$ with $5 \% \mathrm{CO}_{2}$. miRNA mimics/inhibitors and negative control (NC) were synthesized by Guangzhou RiboBio Co. Ltd (Guangzhou, China), and sequences are presented in Table I. Following starvation in serum-free medium for 12 h, U87 cells were transfected with miR-7, miR-181 and miR-195 mimics, miR-15a, miR-16, miR-20a, miR-21, miR-26a andmiR-222 inhibitors, or NC using Xtreme GENE siRNA transfection reagent (Roche Diagnostics, Basel, Switzerland). Transfection concentrations ranged between 10 and $50 \mathrm{nM}$.

Reverse transcription-quantitative polymerase chain reaction $(R T-q P C R)$. Total RNA was extracted from normal brain tissue and U87 cells using TRIzol reagent (Invitrogen; Thermo Fisher Scientific, Inc.) according to the manufacturer's protocol. A total of $0.5 \mu \mathrm{g}$ RNA was reverse transcribed into first strand cDNA using a ReverTraAce ${ }^{\circledR}$ qPCR RT kit (Toyobo Co., Ltd, Osaka, Japan) in a $10 \mu \mathrm{l}$ reaction mixture containing the following: Total RNA $(0.5 \mu \mathrm{g}), 5 \mathrm{X}$ RT buffer $(2 \mu \mathrm{l})$, RT Enzyme Mix $(0.5 \mu \mathrm{l})$, Primer Mix $(0.5 \mu \mathrm{l})$, made up to $10 \mu \mathrm{l}$ with Nuclease-free water. The RT reaction was performed as follows: $25^{\circ} \mathrm{C}$ for $10 \mathrm{~min}, 37^{\circ} \mathrm{C}$ for $120 \mathrm{~min}, 85^{\circ} \mathrm{C}$ for $5 \mathrm{~min}$ and termination at $4^{\circ} \mathrm{C}$ for $5 \mathrm{~min}$. The expression levels of miR-15a, miR-16, miR-20a, miR-21, miR-26a, miR-222, miR-7, miR-181 and miR-195 were determined using SYBR Green Mix (Invitrogen; Thermo Fisher Scientific, Inc.) on an ABI 7500 Fast Real-Time PCR system (Applied Biosystems;
Table I. Sequences of the miRNA mimics and inhibitors.

A, Sequences of miRNA inhibitors

\begin{tabular}{lc}
\hline miRNA & \multicolumn{1}{c}{ Sequence } \\
\hline Hsa-miR-21 & UAGCUUAUCAGACUGAUGUUGA \\
Hsa-miR-20a & UAAAGUGCUUAUAGUGCAGGUAG \\
Hsa-miR-15a & UAGCAGCACAUAAUGGUUUGUG \\
Hsa-miR-16 & UAGCAGCACGUAAAUAUUGGCG \\
Hsa-miR-26 & UUCAAGUAAUCCAGGAUAGGCU \\
Hsa-miR-222 & CUCAGUAGCCAGUGUAGAUCCU \\
\hline
\end{tabular}

$\mathrm{B}$, Sequences of miRNA mimics

miRNA Sequence

$\begin{array}{ll}\text { Hsa-miR-7 } & \text { UGGAAGACUAGUGAUUUUGUUGU } \\ \text { Hsa-miR-181 } & \text { AACAUUCAACGCUGUCGGUGAGU } \\ \text { Hsa-miR-195 } & \text { UAGCAGCACAGAAAUAUUGGC }\end{array}$

$\mathrm{C}$, Sequence of negative control

\begin{tabular}{lc}
\hline miRNA & Sequence \\
\hline cel-miR-239b & UUUGUACUACACAAAAGUACUG
\end{tabular}

miRNA, microRNA; miR, miRNA.

Thermo Fisher Scientific, Inc.). The PCR conditions used to detect the miRNAs were as follows: $95^{\circ} \mathrm{C}$ for $10 \mathrm{~min}$ and 35 cycles at $95^{\circ} \mathrm{C}$ for $15 \mathrm{sec}$ and $60^{\circ} \mathrm{C}$ for $1 \mathrm{~min}$. The miRNA expression relative to U6 was calculated using the $2^{-\Delta \Delta} \mathrm{Cq}$ method (22). The primers for amplification of U6, miR-15a, miR-16, miR-21, miR-20a, miR-26a, miR-7, miR-181, miR-222 and miR-195 are presented in Table II.

Cell viability assay. U87 cells were cultured in 96-well culture plates at a density of $5 \times 10^{4}$ cells/well. Cell viability was assessed by measuring mitochondrial dehydrogenase activity. Following miRNA transfection, U87 cells were incubated with $10 \mu \mathrm{l}$ MTT $(0.5 \mathrm{mg} / \mathrm{ml}$; Sigma-Aldrich; Merck KGaA, Darmstadt, Germany) at $37^{\circ} \mathrm{C}$ for $4 \mathrm{~h}$. The purple formazan crystals were dissolved in $150 \mu 1$ dimethylsulfoxide. The absorbance was measured using a spectrophotometer (Tecan Group, Ltd., Männedorf, Switzerland) at $490 \mathrm{~nm}$.

Terminal deoxynucleotidyl-transferase-mediated dUTP nick end labeling (TUNEL) staining assay. TUNEL staining was detected using an in situ cell death detection kit (Roche Diagnostics) according to the manufacturer's protocol. Following three washes with PBS, U87 cells $\left(2-5 \times 10^{6}\right)$ were fixed with $4 \%$ paraformaldehyde at room temperature and permeabilized in $0.1 \%$ Triton X-100 sodium citrate buffer on ice. The kits were used to label apoptotic cells, and the nuclei were stained with DAPI. The numbers of total cells and TUNEL-positive cells were automatically counted using 
Table II. Primers for reverse transcription-quantitative polymerase chain reaction.

Primers

\begin{tabular}{|c|c|c|}
\hline $\operatorname{miR}$ & Forward & Reverse \\
\hline miR-21 & GCGGCGGTAGCTTATCAGACTG & TAGCTTATCAGACTGATGTTGA \\
\hline miR-20a & GCGGCGGTAAAGTGCTTATAGTG & TAAAGTGCTTATAGTGCAGGTAG \\
\hline miR-15a & GCGGCGGTAGCAGCACATAATG & TAGCAGCACATAATGGTTTGTG \\
\hline $\operatorname{miR}-16$ & GCGGCGGTAGCAGCACGTAAAT & TAGCAGCACGTAAATATTGGCG \\
\hline miR-26a & GCGGCGGTTCAAGTAATCCAGG & TTCAAGTAATCCAGGATAGGCT \\
\hline $\operatorname{miR}-222$ & GCGGCGGACCTGGCATACAATG & ACCTGGCATACAATGTAGATTT \\
\hline miR-7 & GCGGCGGTGGAAGACTAGTG & TGGAAGACTAGTGATTTTGTTGT \\
\hline miR-181 & GCGGCGGAACATTCAACGCTGTC & AACATTCAACGCTGTCGGTGAGT \\
\hline miR-195 & GCGGCGGTAGCAGCACAGAAAT & TAGCAGCACAGAAATATTGGC \\
\hline U6 & GCTTCGGCAGCACATATACTAAAAT & CGCTTCACGAATTTGCGTGTCAT \\
\hline
\end{tabular}

miR, microRNA.

Image-Pro Plus v6.0 software (Media Cybernetics, Inc., Rockville, MD, USA). The apoptosis rate was defined as the ratio of apoptotic cells to total cells.

Detection of apoptosis by flow cytometry. Cell apoptosis was assayed using annexin V-fluorescein isothiocyanate (FITC) /propidium iodide (PI) using the Annexin V-FITC Cell Apoptosis Detection kit (Beyotime Institute of Biotechnology) according to the manufacturer's protocol. Cells $\left(2-3 \times 10^{5}\right.$ cells/well) were cultured in 6 -well plates. Following the various indicated transfection treatments, the cells were digested with trypsin and collected. Cells were washed twice with ice-cold PBS and stained with annexin V-FITC for $12 \mathrm{~min}$ and PI for $5 \mathrm{~min}$. The results were analyzed with CellQuest software v5.1 (BD Biosciences, Franklin Lakes, NJ, USA) and expressed as the percentage of apoptotic cells at the early stage (annexin V-FITC+/PI-; Q4) and the late stage (annexin V-FITC+/PI+; Q2).

Heat map analysis. MATLAB software (vR2012b; MathWorks, Inc., Natick, MA, USA) was used to produce heat maps. Relative cell viability data was inputted and calculated using the surf function to obtain a three-dimensional colored surface model.

Statistical analyses. GraphPad Prism v6.0 software (GraphPad Software, Inc. La Jolla, CA, USA) was used for statistical analysis. All data are expressed as the mean \pm standard error of the mean. Statistical analysis was performed using one-way analysis of variance (ANOVA) followed by Bonferroni's test. $\mathrm{P}<0.05$ was considered to indicate a statistically significant difference.

\section{Results}

miRNAs are aberrantly expressed in U87 cells. Based on previous reports demonstrating aberrant expression of miRNAs in glioma cells, the expression levels of nine miRNAs in U87 cells and normal brain tissue were examined using
RT-qPCR. Compared with the expression levels of miRNAs in normal tissue, miR-15a, miR-16, miR-20a, miR-21, miR-26a, and miR-222 were upregulated in U87 cells by 5.8-, 5.0-, 6.2-, 6.4-, 7.3-, and 6.0-fold, respectively. By contrast, miR-7, miR-181, and miR-195 were downregulated. miR-21 exhibited the most marked alteration among the six miRNAs, exhibiting increased expression in U87 cells. The expression levels of the three downregulated miRNAs exhibited similar rates of reduction, with an average relative expression level of $\sim 0.35$ (Fig. 1).

Effect of miRNAs on the cell viability of U87 cells. U87 cells were transfected with miRNA inhibitors and miRNA mimics at 30 and $50 \mathrm{nM}$, and their effects on the U87 cells were investigated using cell viability assays (Fig. 2). The results of the present study demonstrated that miR-21 and miR-26a inhibitors exhibited effects on the viability of U87 cells when used at $30 \mathrm{nM}$; as the transfection concentration increased to $50 \mathrm{nM}$, the inhibitory effects of the miRNA inhibitors and mimics on cell viability were markedly increased.

Synergistic effects of miRNAs on the viability of U87 cells. In order to identify miRNA combinations with synergistic effects, the above-described miRNAs were investigated as miRNA-miRNA pairs according to calculated synergy scores. According to the miRNA transfection concentration test, cells were cotransfected with two miRNA inhibitors (1:1) at $30 \mathrm{nM}$ each, in order to avoid off-target effects induced by high concentrations of miRNAs and to screen out miRNA combinations with potent synergistic effects at low doses. A total of six upregulated miRNAs were divided into miRNA pairs as follows: $\mathrm{miR}-20 \mathrm{a} / 15 \mathrm{a}, \mathrm{miR}-16 / 20 \mathrm{a}, \mathrm{miR}-21 / 16, \mathrm{miR}-21 / 15 \mathrm{a}$, miR-21/20a, miR-16/222, miR-21/222, and miR-21/26, and cells were cotransfected with these miRNA pairs. Compared with the NC group, U87 cells exhibited significantly reduced cell viability following cotransfection with two low-dose miRNAs. Additionally, following cotransfection with miR-20a/15a, miR-16/20a, miR-21/16, miR-21/15a, and miR-21/20a inhibitors, the combined inhibitory effect of each miRNA pair on the viability of U87 cells was increased compared with the 
A

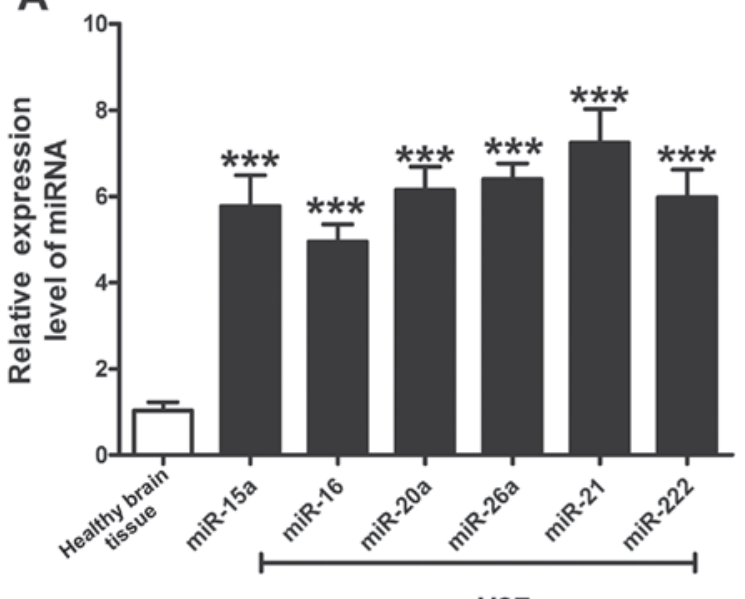

B

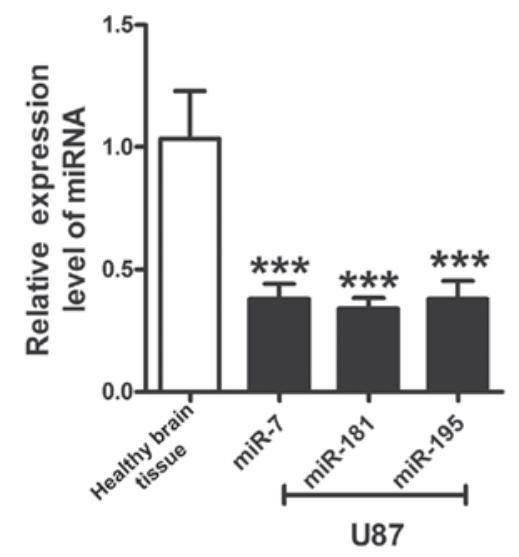

Figure 1. Aberrant expression of miRNAs in U87 cells. (A) Upregulated miRNAs and (B) downregulated miRNAs. $n=4$. ${ }^{* * *} \mathrm{P}<0.001$ vs. healthy brain tissue. miRNA, microRNA.

A

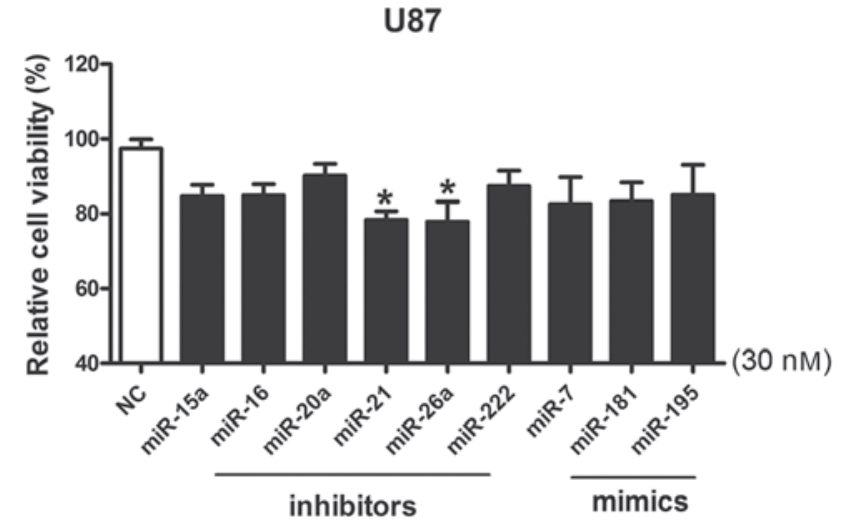

B

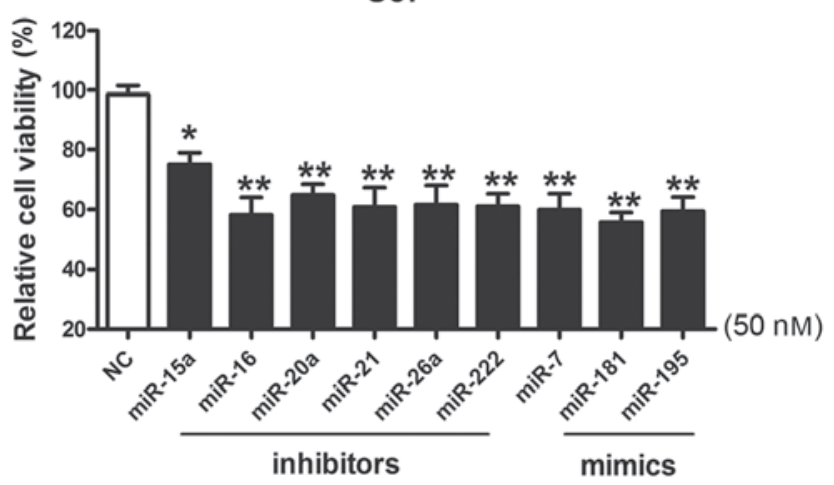

Figure 2. Effects of miRNA inhibitors or mimics on the viability of U87 cells at (A) $30 \mathrm{nM}$ and (B) $50 \mathrm{nM}$. ${ }^{*} \mathrm{P}<0.05$, ${ }^{* *} \mathrm{P}<0.01 \mathrm{vs}$. NC. miRNA, microRNA; $\mathrm{NC}$, negative control.

sum of the individual inhibitory effects of each of the transfected miRNAs. Cell viability was reduced to 77.2 and $88.4 \%$ following transfection with miR-21 inhibitor and miR-20a inhibitor, respectively, whereas viability was $48.5 \%$ following cotransfection with miR-21 and miR-20a inhibitors together, indicating that miR-21 and miR-20a exhibited the most potent synergistic inhibitory effect on the viability of U87 cells (Fig. 3).

Following investigation of the synergism of upregulated miRNAs, downregulated miRNAs, miR-7, miR-181 and miR-195, were investigated as miRNA pairs. It was observed that, although cotransfection with miR-7/181, miR-7/195, and miR-181/195 mimics resulted in marked reductions in cell viability compared with that in the $\mathrm{NC}$ group, the effects were additive rather than synergistic effects (Fig. 4).

Screening of the potent synergistic effects of $m i R-21$ and $m i R-20 a$. Following identification of the potent synergistic effects of miR-21 and miR-20a, the synergistic effects were further investigated at different concentrations and concentration ratios in the range of 10-50 nM. Cell viability assays and heat map analyses demonstrated that with the same transfection concentrations, miR-21 exerted increased effects on cells compared with miR-20a. The miRNA pair of miR-21 (30 $\mathrm{nM})$ and miR-20a (30 $\mathrm{nM})$ exhibited the most marked inhibitory effect on cell viability (Fig. 5).

Synergistic effect of $m i R-21$ and miR-20a cotransfection on apoptosis in U87 cells. In order to further investigate the synergistic effects of miR-21 and miR-20a, TUNEL staining was used for qualitative observation of apoptotic cells and the percentage of apoptotic cells was quantified using Image-Pro Plus software. Compared with the NC group, transfection with a miR-21 inhibitor significantly promoted apoptosis in U87 cells, and transfection with miR-20a promoted apoptosis to a limited degree. By contrast, cotransfection with miR-21 and miR-20a markedly promoted apoptosis with an evident synergistic effect (Fig. 6).

Flow cytometry was performed to quantitatively analyze the types and proportions of apoptotic cells. As presented in Fig. 7, compared with the NC group, in which the apoptotic rate was $9.5 \%$ [quadrant $(\mathrm{Q}) 2,7.0 \%$; Q4, 2.5\%], transfection with miR-21 or miR-20a alone markedly promoted apoptosis 
A
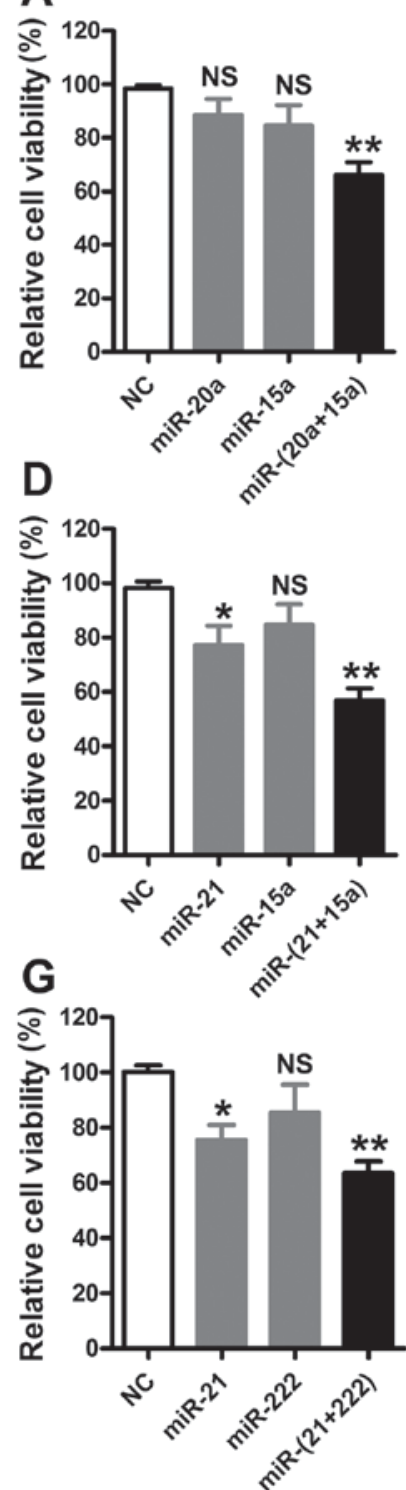

B
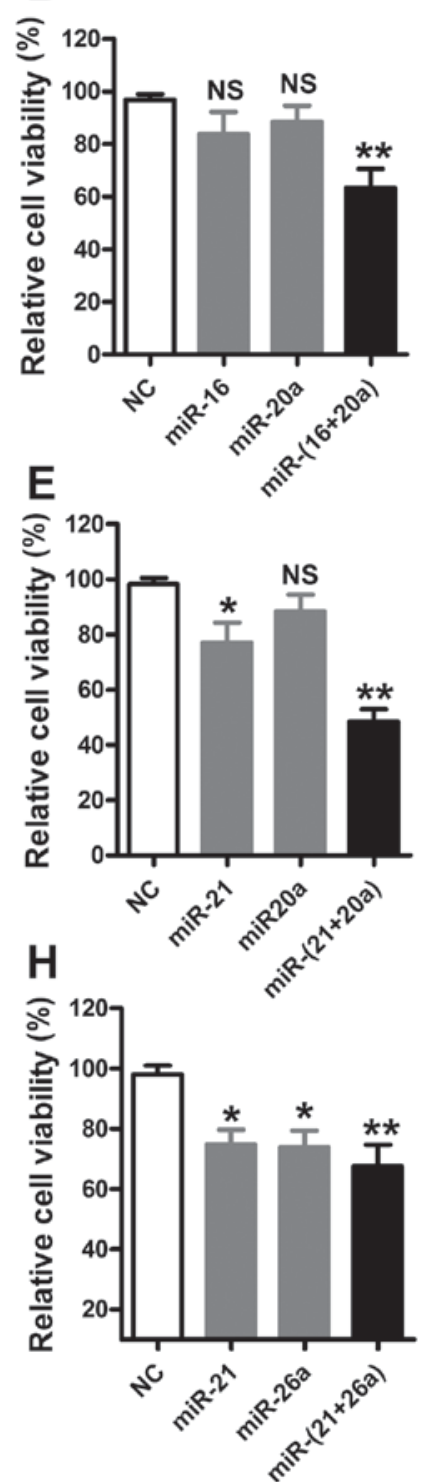

C
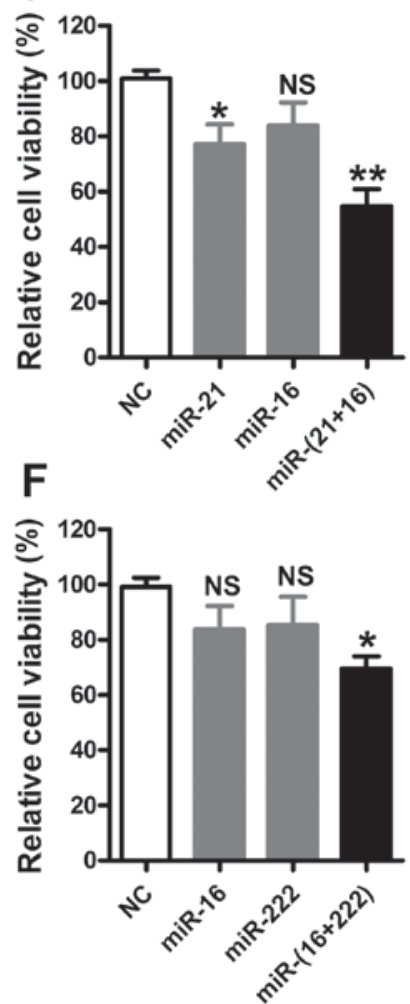

Figure 3. Results of synergistic effects of miRNA inhibitors ( $n=6)$. MTT assays of cells transfected with the miRNA pairs (A) miR-20a:miR-15a, (B) miR-16:miR-20a, (C) miR-21:miR-16, (D) miR-21:miR-15a, (E) miR-21:miR-20a, (F) miR-16:miR-222, (G) miR-21:miR-222 and (H) miR-21:miR-26a. All miRNAs were used at a concentration of $30 \mathrm{nM} .{ }^{*} \mathrm{P}<0.05,{ }^{* *} \mathrm{P}<0.01$ vs. NC. miRNA, microRNA; NC, negative control; NS, not significant.

A

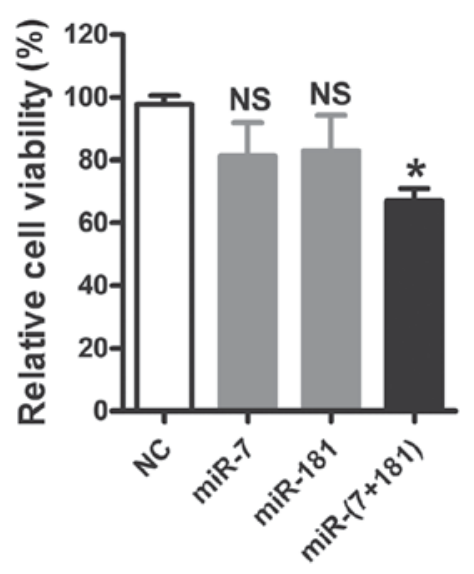

B

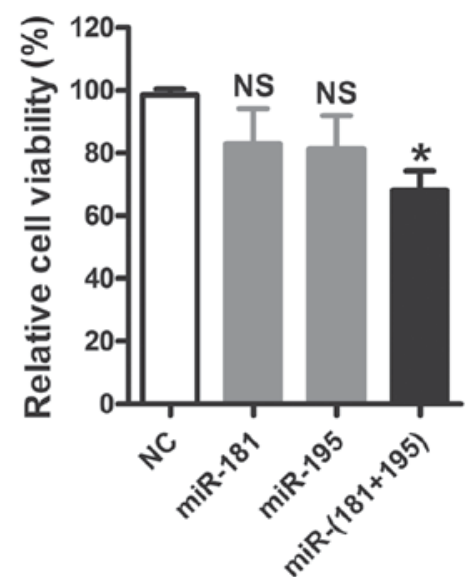

C

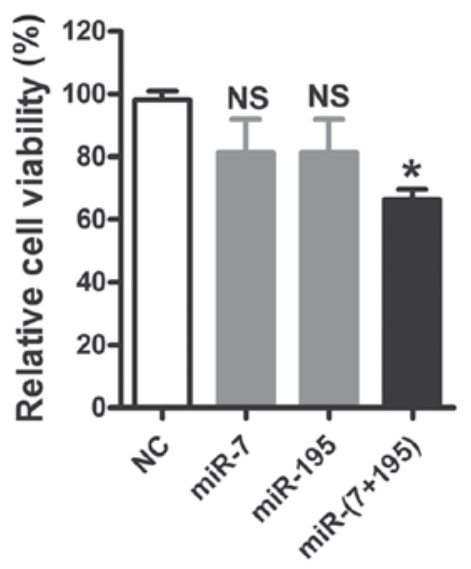

Figure 4. Results of synergistic effects of miRNA mimics ( $n=6$ ). MTT assays of cells transfected with the miRNA pairs (A) miR-7:miR-181,(B) miR-181:miR-195 and (C) miR-7:miR-195. All miRNAs were used at a concentration of $30 \mathrm{nM}$. "P<0.05 vs. NC. miRNA, microRNA; NC, negative control; NS, not significant. 


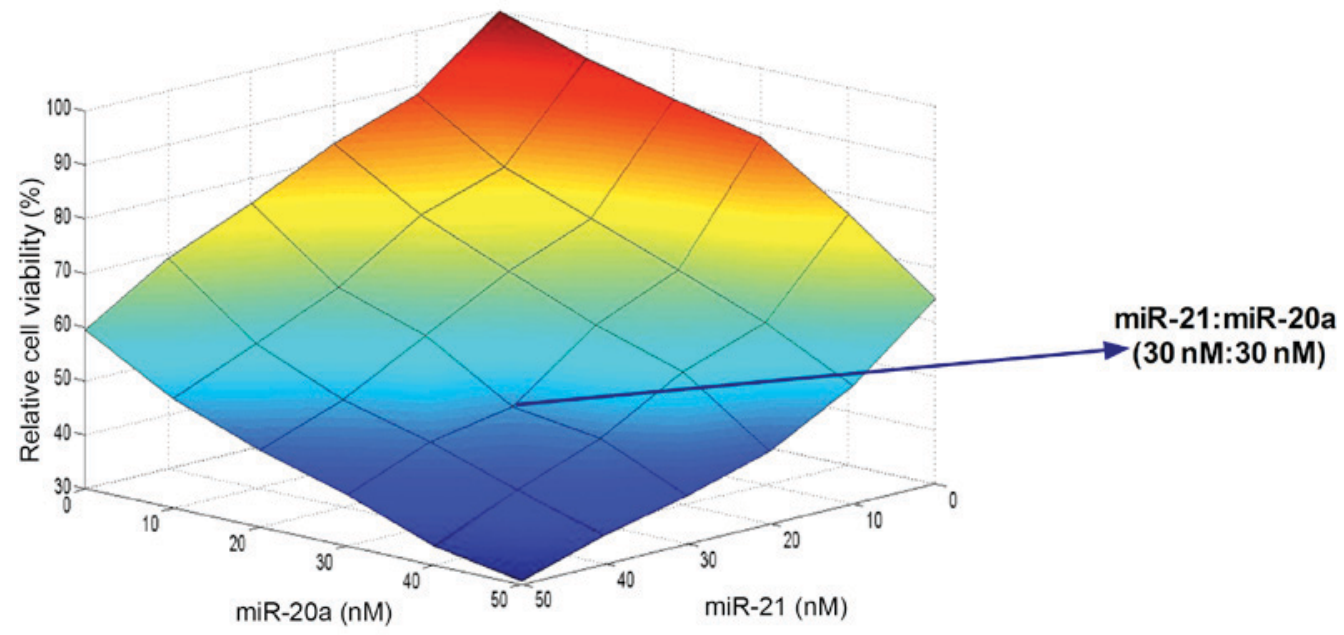

Figure 5. Heat map analysis of the viability of U87 cells transfected with miR-20a inhibitor and miR-21 inhibitor at different transfection concentration ratios. miR, microRNA.

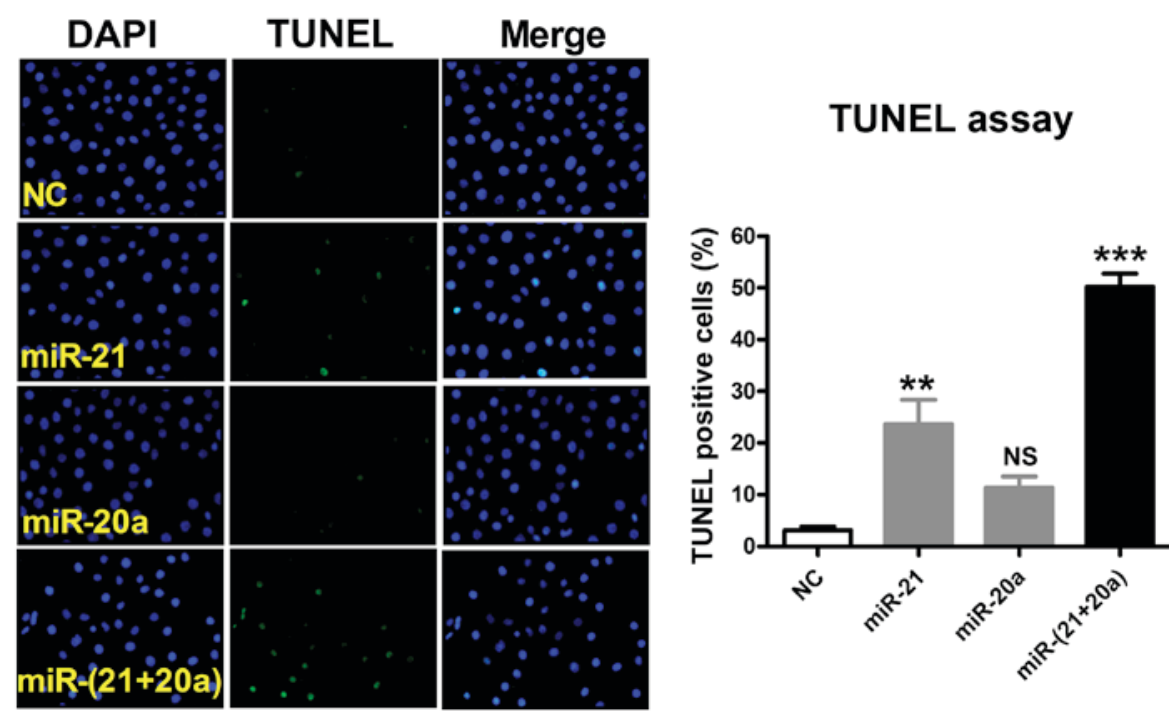

Figure 6. TUNEL assays of synergy between miR-21 and miR-20a $(n=5) .{ }^{* *} \mathrm{P}<0.01,{ }^{* * * *} \mathrm{P}<0.001 \mathrm{vs}$. NC. TUNEL, terminal deoxynucleotidyl-transferase-mediated dUTP nick end labeling; miR; microRNA; NC, negative control.
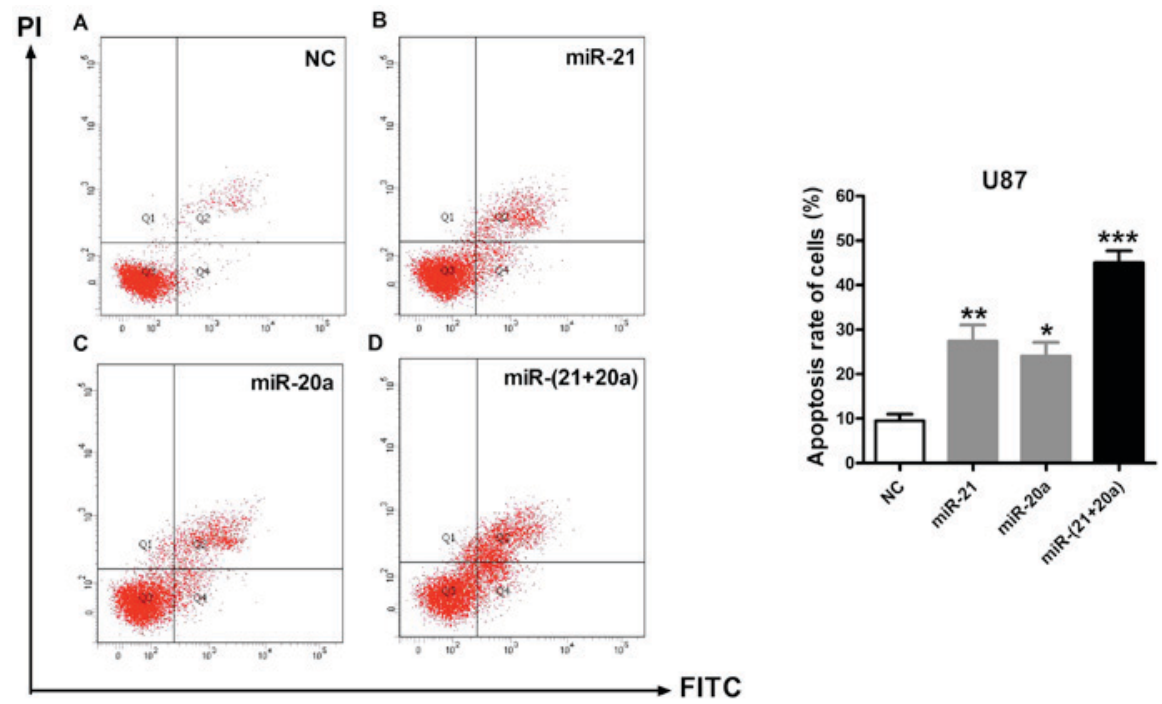

Figure 7. Flow cytometry assays of (A) NC, (B) miR-21, (C) miR-20a and (D) miR-21 combined with miR-20a (n=5). " $\mathrm{P}<0.05,{ }^{* * *} \mathrm{P}<0.01,{ }^{* * *} \mathrm{P}<0.001 \mathrm{vs}$. NC. miR; microRNA; NC, negative control; PI, propidium iodide; FITC, fluorescein isothiocyanate. 
in U87 cells, leading to an average apoptotic rate of $27.4 \%(\mathrm{Q} 2$, 21.7\%; Q4,5.7\%) and 24.0\% (Q2, 17.7\%; Q4,6.3\%), respectively, whereas cotransfection with miR-21 and miR-20a yielded an average apoptotic rate of $45.0 \%$ (Q2, 36.2\%; Q4, 8.8\%). Flow cytometric analysis demonstrated that cotransfection with miR-21 and miR-20a promoted apoptosis, particularly early apoptosis, in U87 cells.

\section{Discussion}

In previous studies, researchers have confirmed the important regulatory effects of miRNAs in glioma and identified aberrantly expressed miRNAs through analysis and validation of miRNAs in blood from tumor tissue and cerebrospinal fluid (23-26). Based on the multi-target regulatory mechanism of miRNAs and long-term study results, it is hypothesized that rational intervention of aberrantly expressed miRNAs may become an important approach to glioma treatment and prevention. Therefore, further analysis of abnormally-expressed miRNAs in brain glioma is of importance to the treatment of glioma.

In the present study, nine abnormal miRNAs in brain glioma cells were selected, and their individual and synergistic effects were examined and compared. The results of the present study demonstrated that the individual regulatory effects of these nine miRNAs on glioma cells are consistent with the results of previous studies (13,27-30). Additionally, the preliminary systems biology approach in the present study indicated that combinations of miRNAs exhibited certain synergistic regulatory effects. The synergistic regulatory effects of miRNAs were experimentally confirmed at the cellular level, and the results were consistent with the results of bioinformatic predictions (20). The present study demonstrated that miR-21 and miR-20a exhibited the largest synergistic effects among all the tested miRNA pairs; the combination of low-concentration (30 nM) miR-21 and miR-20a inhibitors promoted apoptosis in U87 cells, increased the ratio of early and late apoptotic cells, and exerted combined effects that were increased compared with the sum of the individual effects.

The synergistic effects of miRNAs have been demonstrated in the treatment of breast cancer and non-small cell lung cancer (NSCLC). Kasinski et al (31) demonstrated that the combined application of miR-34a and let-7miR precursor mimics was able to improve the efficacy of treatment for NSCLC, and Stahlhut and Slack (32) demonstrated that it was able to further enhance the sensitivity of NSCLC to erlotinib treatment. Devulapally et al (33) demonstrated that the combined use of miR-21 and miR-10b inhibitors was able to increase the inhibitory effects of the miRNAs on breast cancer cells and promote cancer cell apoptosis. These previous experimental results are consistent with the results of the present study. In addition to the synergistic interference of miRNAs in tumor cells, synergistic regulatory effects of miRNAs have been observed in the regulation of cardiovascular diseases (20).

Compared with single drug administration targeting a single miRNA, selecting two low-dose miRNAs for synergistic interference has advantages. Synergistic interference is able to increase the effects of the treatment and, additionally, reduce the dose of any single miRNA in order to avoid the off-target effects of miRNAs and achieve multi-target, high-efficiency regulatory effects. However, there were limitations to the present study. The analyses were only performed at the cellular level; detailed studies and animal experiments are required in order to determine the mechanisms of miRNA synergistic regulation. Additionally, the miRNA delivery system used in the present study is associated with certain side effects; therefore, in order to achieve targeted and stable interference effects, drug delivery systems and materials suitable for miRNAs need to be developed. In previous studies, encapsulation of miRNAs with high-molecular-weight polymers, including poly lactic-co-glycolic acid and polyethylene glycol, has been observed to increase the in vivo stability and targeting of miRNAs (33-35).

In conclusion, in the present study, it was observed that there exist certain miRNA combinations, which are able to exert synergistic tumor-inhibitory effects on the regulation of apoptosis in brain glioma cells. Of the miRNAs examined, the combined use of miR-21 and miR-20a inhibitors demonstrated the clearest synergistic pro-apoptotic effects. The present study supports the development of rational, effective and novel noncoding RNA-based treatment strategies, for the prevention and treatment of glioma.

\section{Acknowledgements}

The present study was supported by the National Natural Science Foundation of China (grant nos. 81272788, 81472368 and 31301095), the Science Foundation of the Health Department of Heilongjiang Province (grant no. 2014301), the Science Foundation of the First Affiliated Hospital of Harbin University (grant no. 2015B014), and the Harbin medical university scientific research innovation fund (No 2016LCZX68).

\section{References}

1. Gladson CL, Prayson RA and Liu WM: The pathobiology of glioma tumors. Annu Rev Pathol 5: 33-50, 2010.

2. Ostrom QT, Gittleman H, Farah P, Ondracek A, Chen Y, Wolinsky Y, Stroup NE, Kruchko C and Barnholtz-Sloan JS: CBTRUS statistical report: Primary brain and central nervous system tumors diagnosed in the United States in 2006-2010. Neuro Oncol 15 (Suppl 2): ii1-ii56, 2013.

3. Morgan LL: The epidemiology of glioma in adults: A 'state of the science' review. Neuro Oncol 17: 623-624, 2015.

4. Louis DN, Perry A, Reifenberger G, von Deimling A, Figarella-Branger D, Cavenee WK, Ohgaki H, Wiestler OD, Kleihues P and Ellison DW: The 2016 World Health Organization classification of tumors of the central nervous system: A summary. Acta Neuropathol 131: 803-820, 2016.

5. Hervey-Jumper SL and Berger MS: Maximizing safe resection of low- and high-grade glioma. J Neuro Oncol 130: pp269-282, 2016.

6. Stavrovskaya AA, Shushanov SS and Rybalkina EY: Problems of glioblastoma multiforme drug resistance. Biochemistry (Mosc) 81: 91-100, 2016

7. Zhang C, Li C, Li J, Han J, Shang D, Zhang Y, Zhang W, Yao Q, Han L, Xu Y, et al: Identification of miRNA-mediated core gene module for glioma patient prediction by integrating high-throughput miRNA, mRNA expression and pathway structure. PLoS One 9: e96908, 2014.

8. Adlakha YK and Saini N: Brain microRNAs and insights into biological functions and therapeutic potential of brain enriched miRNA-128. Mol Cancer 13: 33, 2014.

9. Onishi M, Ichikawa T, Kurozumi K and Date I: Angiogenesis and invasion in glioma. Brain Tumor Patho 28: pp13-24, 2011. 
10. Gaur AB, Holbeck SL, Colburn NH and Israel MA: Downregulation of Pdcd4 by mir-21 facilitates glioblastoma proliferation in vivo. Neuro Oncol 13: 580-590, 2011.

11. Li Y, Zhao S, Zhen Y, Li Q, Teng L, Asai A and Kawamoto K: A miR-21 inhibitor enhances apoptosis and reduces G(2)-M accumulation induced by ionizing radiation in human glioblastoma U251 cells. Brain Tumor Pathol 28: 209-214, 2011.

12. Zhang C, Zhang J, Hao J, Shi Z, Wang Y, Han L, Yu S, You Y, Jiang T, Wang J, et al: High level of miR-221/222 confers increased cell invasion and poor prognosis in glioma. J Transl Med 10: e119, 2012.

13. Ernst A, Campos B, Meier J, Devens F, Liesenberg F, Wolter M, Reifenberger G, Herold-Mende C, Lichter P and Radlwimmer B: De-repression of CTGF via the miR-17-92 cluster upon differentiation of human glioblastoma spheroid cultures. Oncogene 29: 3411-3422, 2010

14. Costa PM, Cardoso AL, Mano M and de Lima MC: MicroRNAs in glioblastoma: Role in pathogenesis and opportunities for targeted therapies. CNS Neurol Disord Drug Targets 14: 222-238, 2015.

15. Wu DG, Wang YY, Fan LG, Luo H, Han B, Sun LH, Wang XF, Zhang JX, Cao L, Wang XR, et al: MicroRNA-7 regulates glioblastoma cell invasion via targeting focal adhesion kinase expression. Chin Med J (Engl) 124: 2616-2621, 2011.

16. Chen G, Zhu W, Shi D, Lv L, Zhang C, Liu P and $\mathrm{Hu}$ W: MicroRNA-181a sensitizes human malignant glioma U87MG cells to radiation by targeting Bcl-2. Oncol Rep 23: 997-1003, 2010.

17. Gabriely G, Wurdinger T, Kesari S, Esau CC, Burchard J, Linsley PS and Krichevsky AM: MicroRNA 21 promotes glioma invasion by targeting matrix metalloproteinase regulators. Mol Cell Biol 28: 5369-5380, 2008.

18. Xu J, Li CX, Li YS, Lv JY, Ma Y, Shao TT, Xu LD, Wang YY, Du L, Zhang YP, et al: MiRNA-miRNA synergistic network: Construction via co-regulating functional modules and disease miRNA topological features. Nucleic Acids Res 39: 825-836, 2011.

19. Hu S, Huang M, Nguyen PK, Gong Y, Li Z, Jia F, Lan F, Liu J, Nag D, Robbins RC and Wu JC: Novel microRNA prosurvival cocktail for improving engraftment and function of cardiac progenitor cell transplantation. Circulation 124 (11 Suppl): S27-S34, 2011

20. Zhu W, Zhao Y, Xu Y, Sun Y, Wang Z, Yuan W and Du Z: Dissection of protein interactomics highlights microRNA synergy. PLoS One 8: e63342, 2013.

21. Singh S, Narang AS and Mahato RI: Subcellular fate and off-target effects of siRNA, shRNA, and miRNA. Pharm Res 28: 2996-3015, 2011.

22. Livak KJ and Schmittgen TD: Analysis of relative gene expression data using real-time quantitative PCR and the 2(-Delta Delta C(T)) Method. Methods 25: 402-408, 2001.

23. Zhang Y, Dutta A and Abounader R: The role of microRNAs in glioma initiation and progression. Front Biosci (Landmark Ed) 17: 700-712, 2012
24. Codo P, Weller M, Meister G, Szabo E, Steinle A, Wolter M, Reifenberger G and Roth P: MicroRNA mediated down-regulation of NKG2D ligands contributes to glioma immune escape. Oncotarget 5: 7651-7662, 2014.

25. Visani M, de Biase D, Marucci G, Cerasoli S, Nigrisoli E, Bacchi Reggiani ML, Albani F, Baruzzi A and Pession A; PERNO study group: Expression of 19 microRNAs in glioblastoma and comparison with other brain neoplasia of grades I-III. Mol Oncol 8: 417-430, 2014.

26. Feng SY, Dong CG, Wu K, Wang XG, Qiao J and Shao JF: Lentiviral expression of anti-microRNAs targeting miR-27a inhibits proliferation and invasiveness of U87 glioma cells. Mol Med Rep 6: 275-281, 2012.

27. Shi R, Wang PY, Li XY, Chen JX, Li Y, Zhang XZ, Zhang CG, Jiang T, Li WB, Ding W and Cheng SJ: Exosomal levels of miRNA-21 from cerebrospinal fluids associated with poor prognosis and tumor recurrence of glioma patients. Oncotarget 6: 26971-26981, 2015.

28. Gwak HS, Kim TH, Jo GH, Kim YJ, Kwak HJ, Kim JH, Yin J, Yoo H, Lee SH and Park JB: Silencing of microRNA-21 confers radio-sensitivity through inhibition of the PI3K/AKT pathway and enhancing autophagy in malignant glioma cell lines. PLoS One 7: e47449, 2012.

29. Han L, Yue X, Zhou X, Lan FM, You G, Zhang W, Zhang KL, Zhang CZ, Cheng JQ, Yu SZ, et al: MicroRNA-21 expression is regulated by $\beta$-catenin/STAT3 pathway and promotes glioma cell invasion by direct targeting RECK. CNS Neurosci Ther 18: 573-583, 2012.

30. Yao X, Li W, Wang Y, Ding Z, Hou W, Zeng M, Deng G, Zhang J and Yang H: Expression of hsa-miR-20a in human glioma tissues and its effect on the proliferation of human glioma cells in vitro. Nan Fang Yi Ke Da Xue Xue Bao 32: 198-201, 2012 (In Chinese).

31. Kasinski AL, Kelnar K, Stahlhut C, Orellana E, Zhao J, Shimer E, Dysart S, Chen X, Bader AG and Slack FJ: A combinatorial microRNA therapeutics approach to suppressing non-small cell lung cancer. Oncogene 34: 3547-3555, 2015.

32. Stahlhut C and Slack FJ: Combinatorial action of MicroRNAs let-7 and miR-34 Effectively synergizes with erlotinib to suppress non-small cell lung cancer cell proliferation. Cell Cycle 14: 2171-2180, 2015.

33. Devulapally R, Sekar NM, Sekar TV, Foygel K, Massoud TF, Willmann JK and Paulmurugan R: Polymer nanoparticles mediated codelivery of antimiR-10b and antimiR-21 for achieving triple negative breast cancer therapy. ACS Nano 9: 2290-2302, 2015.

34. Jin H, Yu Y, Chrisler WB, Xiong Y, Hu D and Lei C: Delivery of MicroRNA-10b with polylysine nanoparticles for inhibition of breast cancer cell wound healing. Breast Cancer (Auckl) 6: 9-19, 2012.

35. Fernandez-Fernandez A, Manchanda R and McGoron AJ: Theranostic applications of nanomaterials in cancer: Drug delivery, image-guided therapy, and multifunctional platforms. Appl Biochem Biotechnol 165: 1628-1651, 2011. 